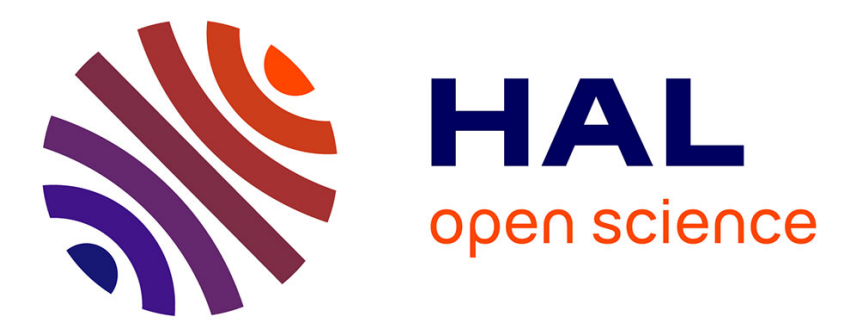

\title{
Coordination and Concurrency in Multi-engine Prolog
} Paul Tarau

\section{To cite this version:}

Paul Tarau. Coordination and Concurrency in Multi-engine Prolog. 13th Conference on Coordination Models and Languages (COORDINATION), Jun 2011, Reykjavik, Iceland. pp.157-171, 10.1007/9783-642-21464-6_11. hal-01582990

\section{HAL Id: hal-01582990 \\ https://hal.inria.fr/hal-01582990}

Submitted on 6 Sep 2017

HAL is a multi-disciplinary open access archive for the deposit and dissemination of scientific research documents, whether they are published or not. The documents may come from teaching and research institutions in France or abroad, or from public or private research centers.
L'archive ouverte pluridisciplinaire HAL, est destinée au dépôt et à la diffusion de documents scientifiques de niveau recherche, publiés ou non, émanant des établissements d'enseignement et de recherche français ou étrangers, des laboratoires publics ou privés. 


\title{
Coordination and Concurrency in Multi-Engine Prolog
}

\author{
Paul Tarau \\ Department of Computer Science and Engineering \\ University of North Texas \\ tarau@cs.unt.edu
}

\begin{abstract}
We discuss the impact of the separation of logic engines (independent logic processing units) and multi-threading on the design of coordination mechanisms for a Prolog based agent infrastructure.

We advocate a combination of coroutining constructs with focus on expressiveness and a simplified, multi-threading API that ensures optimal use available parallelism.

In this context, native multi-threading is made available to the application programmer as a set of high-level primitives with a declarative flavor while cooperative constructs provide efficient and predictable coordination mechanisms. As illustrations of our techniques, a parallel fold operation as well as cooperative implementations of Linda blackboards and publish/subscribe are described.

Keywords: multi-engine Prolog, agent coordination, high-level multithreading, coroutining Linda blackboards, publish/subscribe, Java-based Prolog system
\end{abstract}

\section{Introduction}

Multi-threading has been adopted in today's Prolog implementations as it became widely available in implementation languages like $\mathrm{C}$ or Java.

An advantage of multi-threading over more declarative concurrency models like various AND-parallel and OR-parallel execution schemes, is that it maps to the underlying hardware directly: on typical multi-core machines threads and processes are mapped to distinct CPUs ${ }^{1}$. Another advantage is that a procedural multi-threading API can tightly control thread creation and thread reuse.

On the other hand, the explicit use of a procedural multi-threading API breaks the declarative simplicity of the execution model of logic based languages. At the same time it opens a Pandora's box of timing and execution order dependencies, resulting in performance overheads for various runtime structures that need to be synchronized. It also elevates risks of software failure due to programmer errors given the mismatch between assumptions about behavior expected

\footnotetext{
${ }^{1}$ We use the word CPU in accordance of what the underlying runtime system and operating system sees as independent processing units in a multi-core/multi-processor machine. For instance, on a two Xeon processor Quad-Core MacPro with hyperthreading, the Java VM sees 16 independent processing units.
} 
to follow the declarative semantics of the core language and the requirements of a procedural multi-threading API.

In this paper we will describe how efficient and flexible agent coordination is facilitated by a design emphasizing the decoupling of the multi-threading API and the logic engine operations and encapsulation of multi-threading in a set of high-level primitives with a declarative flavor.

In this process, we use threads encapsulated as high level programming language constructs with focus on performance benefits, and we are resorting to determinacy, through lightweight, cooperative sequential constructs, to express coordination patterns.

We have implemented the API in the context of an experimental, Java-based Prolog system, Lean-Prolog ${ }^{2}$.

LeanProlog is based on a reimplementation of BinProlog's virtual machine, the BinWAM. It succeeds our Jinni Prolog implementation that has been used in various applications [1-4] as an intelligent agent infrastructure, by taking advantage of Prolog's knowledge processing capabilities in combination with a simple and easily extensible runtime kernel supporting a flexible reflexion mechanism. Naturally, this has suggested to investigate whether some basic agent-oriented language design ideas can be used for a refactoring of Prolog's interoperation with the external world, including interaction with other instances of the Prolog processor itself.

Agent programming constructs have influenced design patterns at "macro level", ranging from interactive Web services to mixed initiative computer human interaction. From the very beginning, Performatives in Agent communication languages $[5,6]$ have made these constructs reflect explicitly the intentionality, as well as the negotiation process involved in agent interactions. At the same time, it has been a long tradition of logic programming languages [7] to use multiple logic engines for supporting concurrent execution.

In this context we have centered our implementation around logic engine constructs providing an API that supports reentrant instances of the language processor. This has naturally led to a view of logic engines as instances of a generalized family of iterators called Fluents [8], that have allowed the separation of the first-class language interpreters from the multi-threading mechanism, while providing a very concise source-level reconstruction of Prolog's built-ins. Later we have extended the original Fluents with a few new operations [9] supporting bi-directional, mixed-initiative exchanges between engines, bringing them closer to an agent-oriented view as autonomous logic processors.

The resulting language constructs, that we have called Interactors, express control, metaprogramming and interoperation with stateful objects and external services.

\footnotetext{
${ }^{2}$ It is called Lean-Prolog as we have consistently tried to keep implementation complexity under control and follow minimalist choices in the design of built-ins, external language interfaces and a layered, modular extension mechanism.
} 
On the other hand, our multi-threading layer has been designed to be independent of the interactor API. This allows assumptions of determinacy when working with multiple engines (and other sequential interactors) within a thread.

The multi-threading API integrates thread-construction with interactors called Hubs that provide synchronization between multiple consumers and producers. It supports high-level performance-centered concurrency patterns while removing the possibility of programming errors involving explicit synchronization.

The guiding architectural principle we based our design on, can be stated concisely as follows: separate concurrency for performance from concurrency for expressiveness. Arguably, it is a good fit with the general idea behind declarative programming languages - delegate as much low level detail to underlying implementation as possible rather than burdening the programmer with complex control constructs.

The paper is organized as follows.

Section 2 overviews logic engines and describes their basic operations and the interactor API that extends the same view to various other built-in predicates. Section 3 introduces Hubs - flexible synchronization devices that allow interoperation and coordination between threads. Section 4 describes a set of high-level multi-threading operations that ensure concurrent execution seen as a means to accelerate computations while keeping the semantics as close as possible to a declarative interpretation.

Sections 5 and 6 show that fundamental coordination patterns like Linda blackboards and publish/subscribe can be implemented cooperatively in terms of sequential operations on logic engines.

Finally, section 7 discusses related work and section 8 concludes the paper.

\section{Logic engines as answer generators}

Our Interactor API has evolved progressively into a practical Prolog implementation framework starting with [8] and continued with [10] and [9]. We summarize it here and refer to [9] for the details of a semantic description in terms of Horn Clause Logic of various engine operations.

A logic engine is simply a language processor reflected through an API that allows its computations to be controlled interactively from another engine very much the same way a programmer controls Prolog's interactive toplevel loop: launch a new goal, ask for a new answer, interpret it, react to it.

A logic engine can be seen as an instance of the Prolog runtime system implementing LD-resolution [11] on a given clause database, together with a set of built-in operations. The command

new_engine(AnswerPattern, Goal, Interactor)

creates a new logic engine, uniquely identified by Interactor, which shares code with the currently running program and is initialized with Goal as its starting point. AnswerPattern is a term, usually a list of variables occurring in Goal, of which answers returned by the engine will be instances. Note however that 
new_engine/3 acts like a typical constructor, no computations are performed at this point, except for initial allocation of data areas.

\subsection{Iterating over computed answers}

Note that our logic engines are seen, in an object oriented-style, as implementing the interface Interactor. This supports a uniform interaction mechanism with a variety of objects ranging from logic engines to file/socket streams and iterators over external data structures.

The ask_interactor/2 operation is used to retrieve successive answers generated by an Interactor, on demand. It is also responsible for actually triggering computations in the engine. The query

ask_interactor(Interactor, AnswerInstance)

tries to harvest the answer computed from Goal, as an instance of AnswerPattern. If an answer is found, it is returned as the (AnswerInstance), otherwise the atom no is returned. Note that bindings are not propagated to the original Goal or AnswerPattern when ask_interactor/2 retrieves an answer, i.e. AnswerInstance is obtained by first standardizing apart (renaming) the variables in Goal and AnswerPattern, and then backtracking over its alternative answers in a separate Prolog interpreter. Therefore, backtracking in the caller interpreter does not interfere with Interactor's iteration over answers. Backtracking over Interactor's creation point, as such, makes it unreachable and therefore subject to garbage collection. An interactor is stopped with the

stop_interactor (Interactor)

operation, that, in the case of logic engines, allows reclaiming resources held by the engine.

So far, these operations provide a minimal API, powerful enough to switch tasks cooperatively between an engine and its client and emulate key Prolog built-ins like if-then-else and findall [8], as well as typical higher order operations like fold and best_of [9].

\section{$2.2 \quad$ A yield/return operation}

The following operations provide a "mixed-initiative" interaction mechanism, allowing more general data exchanges between an engine and its client.

First, like the yield return construct of $\mathrm{C \#}$ and the yield operation of Ruby and Python, our return/1 operation

return (Term)

will save the state of the engine and transfer control and a result Term to its client. The client will receive a copy of Term simply by using its ask_interactor/2 operation.

Note that an interactor returns control to its client either by calling return/1 or when a computed answer becomes available. By using a sequence of return 
and ask_interactor operations, an engine can provide a stream of intermediate/final results to its client, without having to backtrack. This mechanism is powerful enough to implement a complete exception handling mechanism simply by defining

throw(E) :- return(exception(E)).

When combined with a catch (Goal, Exception, OnException), on the client side, the client can decide, upon reading the exception with ask_interactor/2, if it wants to handle it or to throw it to the next level.

\subsection{Coroutining logic engines}

Coroutining has been in use in Prolog systems mostly to implement constraint programming extensions. The typical mechanism involves attributed variables holding suspended goals that may be triggered by changes in the instantiation state of the variables. We discuss here a different form of coroutining, induced by the ability to switch back and forth between engines.

The operations described so far allow an engine to return answers from any point in its computation sequence. The next step is to enable an engine's client $^{3}$ to inject new goals (executable data) to an arbitrary inner context of an engine. Two new primitives are needed:

to_engine(Engine, Data)

that is called by the client ${ }^{4}$ to send data to an Engine, and

from_engine(Data)

that is called by the engine to receive a client's Data.

A typical use case for the Interactor API looks as follows:

1. the client creates and initializes a new engine

2. the client triggers a new computation in the engine, parameterized as follows:

(a) the client passes some data and a new goal to the engine and issues an ask_interactor/2 operation that passes control to it

(b) the engine starts a computation from its initial goal or the point where it has been suspended and runs (a copy of) the new goal received from its client

(c) the engine returns (a copy of) the answer, then suspends and returns control to its client

3. the client interprets the answer and proceeds with its next computation step

4. the process is fully reentrant and the client may repeat it from an arbitrary point in its computation

\footnotetext{
3 Another engine, that uses an engine's services.

${ }^{4}$ Equivalently the tell_interactor/2 generic interactor predicate can be also used here.
} 


\section{Hubs and threads}

As a key difference with typical multi-threaded Prolog implementations like Ciao-Prolog [12] and SWI-Prolog [13], our Interactor API is designed up front with a clear separation between engines and threads as we prefer to see them as orthogonal language constructs.

To ensure that communication between logic engines running concurrently is safe and synchronized, we hide the engine handle and provide a producer/consumer data exchanger object, called a Hub, when multi-threading.

A Hub can be seen as an interactor used to synchronize threads. On the Prolog side it is introduced with a constructor hub/1 and works with the standard interactor API:

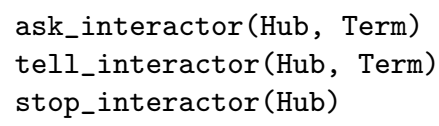

On the Java side, each instance of the Hub class provides a synchronizer between $\mathrm{M}$ producers and $\mathrm{N}$ consumers. A Hub supports data exchanges through a private object port and it implements the Interactor interface. Consumers issue ask_interactor/2 operations that correspond to tell_interactor/2 operations issued by producers.

A group of related threads are created around a Hub that provides both basic synchronization and data exchange services. The built-in

new_logic_thread(Hub, X, G, Clone, Source)

creates a new thread by either "cloning" the current Prolog code and symbol spaces or by loading new Prolog code in a separate name space from a Source (typically a precompiled file or a stream). Usually a default constructor

new_logic_thread(Hub, X, G)

is used. It shares the code but it duplicates the symbol table to allow independent symbol creation and symbol garbage collection to occur safely in multiple threads without the need to synchronize or suspend thread execution.

\section{High-level concurrency with higher-order constructs}

Encapsulating concurrent execution patterns in high-level abstractions, when performance gains are the main reason for using multiple threads, avoids forcing a programmer to suddenly deal with complex procedural issues when working with (mostly) declarative constructs in a language like Prolog. It is also our experience that in an exclusively dynamically-typed language like Prolog this reduces software risks significantly.

One of the deficiencies of sequential or multi-threaded findall-like operations is that they might build large lists of answers unnecessarily. With inspiration drawn from combinators in functional languages, one can implement a more flexible multi-threaded fold operation instead. 
The predicate multi_fold(F, XGs, Xs) runs a list of goals XGs of the form Xs :- G and combines, with $F$, their answers, to accumulate them into a single final result, without building intermediate lists.

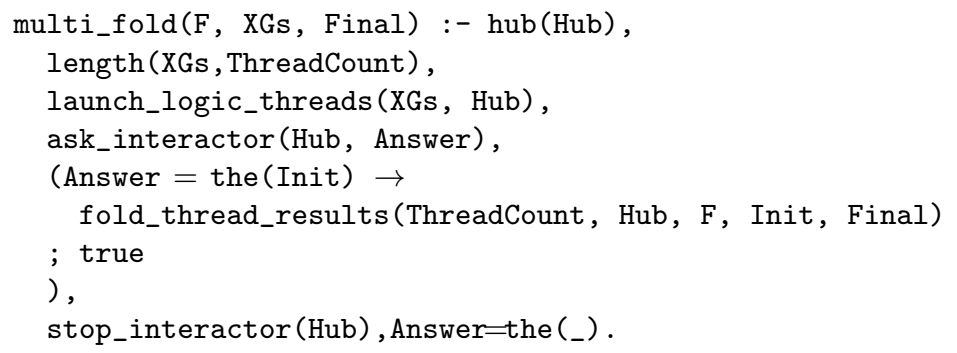

The predicate multi_fold relies on the predicate launch_logic_threads to run threads initiated by the goal list XGs. When launching the threads, we ensure that they share the same Hub for communication and synchronization.

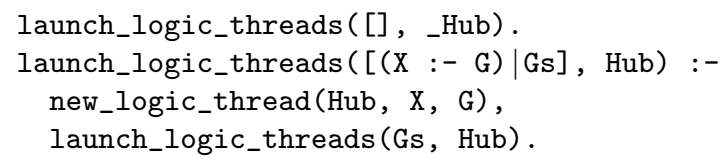

Once all threads are launched, we use the predicate fold_thread_results to collect results computed by various threads from Hub, and to combine them into a single result, while keeping track of the number of threads that have finished their work.

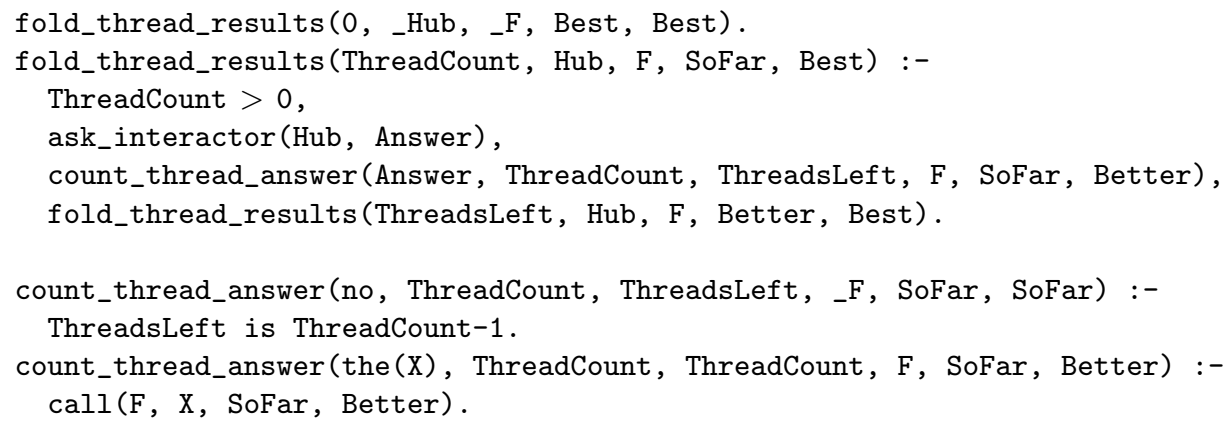

A typical application is the predicate multi_best (F, XGs, M), which runs a list of goals XGs of the form $\mathrm{N}:-\mathrm{G}$ where $\mathrm{N}$ is instantiated to a numeric value. By using max/3 to combine the current best answers with a candidate one it extracts at the the maximum $\mathrm{M}$ of all answers computed (in an arbitrary order) by all threads.

multi_best(XGs,R) :- multi_fold(max, XGs, R) .

Note that, as in the case of its fold cousins in functional languages, multi_fold can be used to emulate various other higher order predicates. For instance a findall-like predicate is emulated as the predicate multi_all(XGs,Xs) which runs a list of goals XGs of the form Xs :- $\mathrm{G}$ and combines all answers to a list using list_cons. 
multi_all(XGs, Rs) :- multi_fold(list_cons, [([] :- true)|XGs], Rs) .

list_cons (X, Xs, $[\mathrm{X} \mid \mathrm{Xs}])$.

A different pattern arises from combinatorial search algorithms where one wants to stop multiple threads as soon as a first solution is found. Things like restarts in SAT solvers and various Monte Carlo algorithms fit in this category.

For instance, the predicate multi_first (K, XGs, Xs) runs each goal of the form Xs :- G on the list XGs, until the first $\mathrm{K}$ answers $\mathrm{Xs}$ are found (or fewer, if less then $\mathrm{K}$ answers exist).

It uses a very simple mechanism built into Lean Prolog's multi-threading API: when a Hub interactor is stopped, all threads associated to it are notified to terminate.

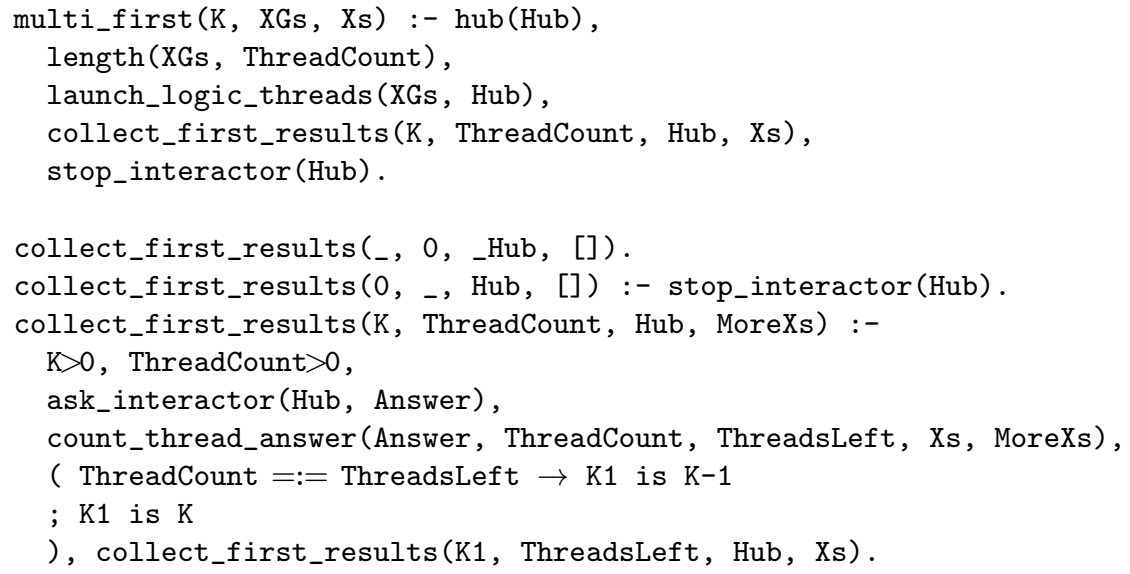

In particular, searching for at most one solution is possible:

multi_first(XGs,X) :- multi_first $(1, \mathrm{XGs},[\mathrm{X}])$.

The multi_first/3 and multi_first/2 predicates provide an alternative to using CUT in Prolog as a means to limit search, while supporting a scalable mechanism for concurrent execution. Note also that multi_first/3 it is more flexible than CUT as it can be used to limit the search to a window of $\mathrm{K}$ solutions. However, in contrast with CUT, the order in which these first solutions are found is arbitrary.

\section{Agent coordination with cooperative Linda blackboards}

The message passing style interaction shown in the previous sections between engines and their clients, can be easily generalized to associative communication through a unification based blackboard interface [14]. Exploring this concept in depth promises more flexible interaction patterns, as out of order operations become possible, matched by association patterns. An interesting question arises at this point. Can blackboard-based coordination be expressed directly in terms of engines, and as such, can it be seen as independent of a multi-threading API? 
We have shown so far that when focusing on performance on multi-core architectures, multi-threading can be encapsulated in high-level constructs that provide its benefits without the need of a complex procedural API.

To support our claim that "concurrency for expressiveness" works quite naturally with coroutining logic engines we will describe here a cooperative implementation of Linda blackboards. In contrast to multi-threaded or multi-process implementations, it ensures atomicity "by design" for various operations. It is an example of concurrency for expressiveness that can be used orthogonally from concurrency for performance to coordinate cooperatively multiple logic engines within a single thread.

The predicate new_coordinator $(\mathrm{Db})$ uses a database parameter $\mathrm{Db}$ (a synthetic name, if given as a free variable, provided by db_ensure_bound) to store the state of the Linda blackboard ${ }^{5}$. The state of the blackboard is described by the dynamic predicates available/1, that keeps track of terms posted by out operations, waiting $/ 2$, that collects pending in operations waiting for matching terms, and running/ 1 , that helps passing control from one engine to the next.

new_coordinator $(\mathrm{Db})$ :- db_ensure_bound (Db),

maplist (db_dynamic(Db), [available/1, waiting/2,running/1]).

The predicate new_task initializes a new coroutining engine, driven by goal G. We shall call such an engine "an agent" in the next paragraphs.

new_task(Db, G) :- new_engine(nothing, (G, fail), E), $\mathrm{db} \_\operatorname{assertz}(\mathrm{Db}$, running $(\mathrm{E}))$.

Three cooperative Linda operations are available to an agent. They are all expressed by returning a specific pattern to the Coordinator.

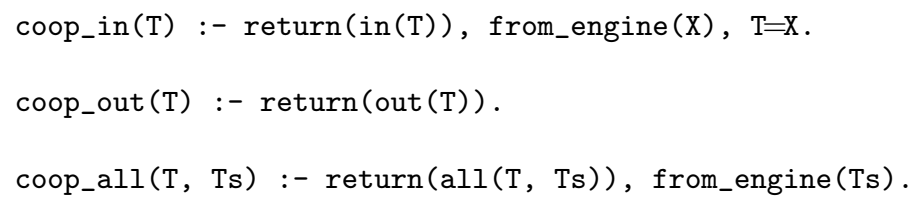

The Coordinator implements a handler for the patterns returned by the agents as follows:

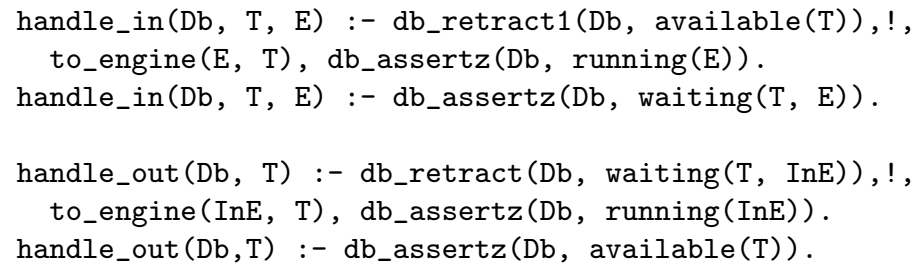

${ }^{5}$ Note, that, as an extension to standard Prolog, Lean Prolog provides multiple dynamic databases, which, in turn, can be emulated, as shown in [9], in terms of logic engines. Their operations (like db_assert/2 similar to assert/1) have an extra first argument that names the database on which they act). 


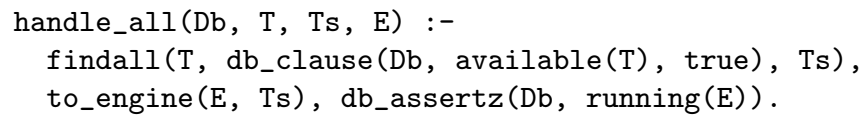

The Coordinator's dispatch loop coordinate/1 (failure driven here to run without requiring garbage collection) works as follows:

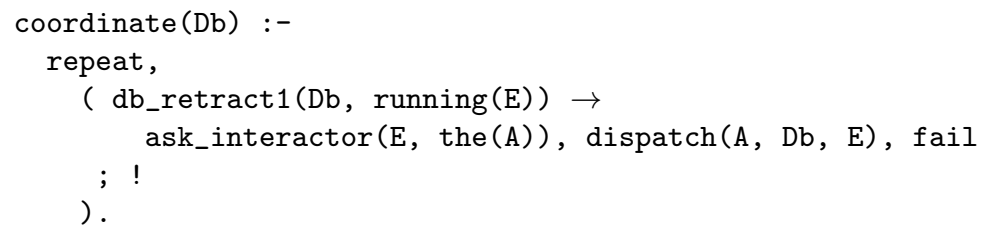

Its dispatch/3 predicate calls the handlers as appropriate.

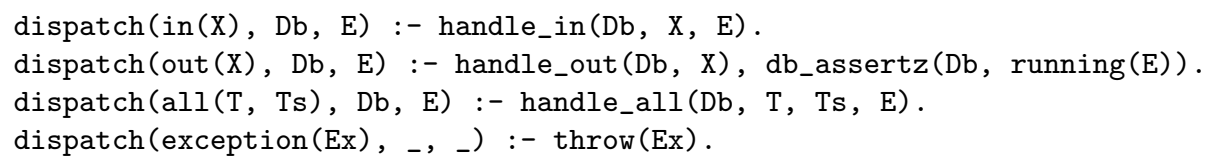

Note also that the predicate dispatch/3 propagates exceptions - in accordance with a "fail fast" design principle.

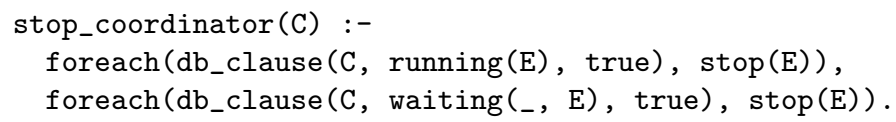

When the coordinator is stopped using stop_coordinator, the database is cleaned of possible records of unfinished tasks in either running or waiting state. This predicate uses a Lean Prolog extension, foreach which makes failure-driven loops more readable - it is defined as follows:

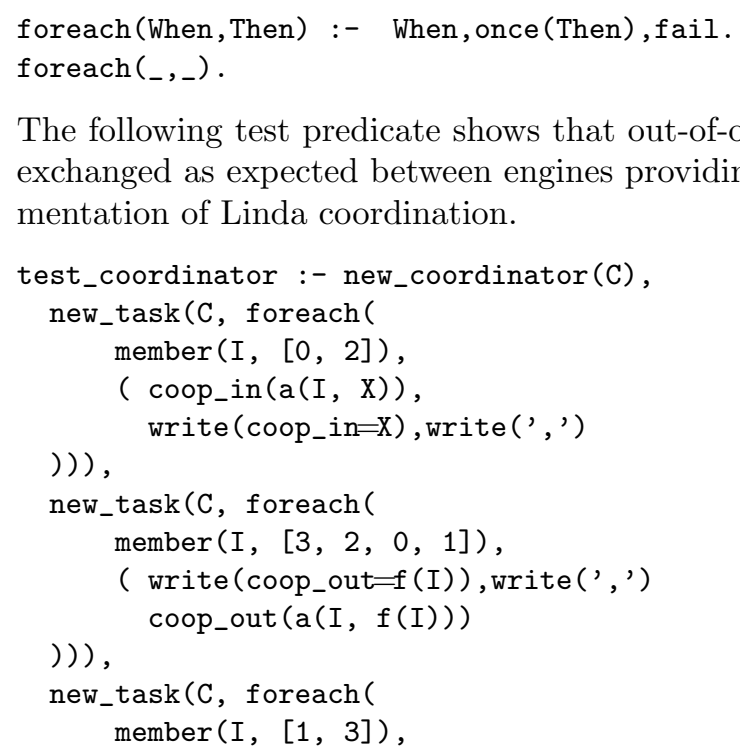
mentation of Linda coordination.

The following test predicate shows that out-of-order in and out operations are exchanged as expected between engines providing a simple transactional imple- 


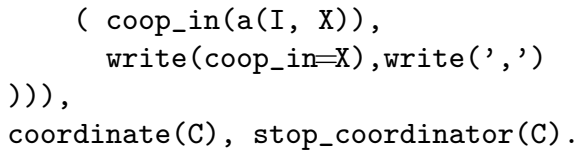

When running the code, one can observe that explicit coroutining control exchanges between engines have been replaced by operations of the higher level Linda coordination protocol.

?- test_coordinator.

coop_out $=f(3)$, coop_out $=f(2)$, coop_out $=f(0)$, coop_in $=f(0)$,

coop_out $=f(1)$, coop_in $=f(2)$, coop_in $=f(1)$, coop_in $=f(3)$.

This shows that "concurrency for expressiveness" in terms of the logic-enginesas-interactors API provides flexible building blocks for the encapsulation of nontrivial high-level concurrency patterns.

\section{Coordinating publishers and subscribers}

We will now describe a cooperative publish/subscribe mechanism that uses multiple dynamic databases and provides, as an interesting feature, associative search through the set of subscribed events.

The predicate publish (Channel, Content) initializes a Channel, implemented as a dynamic database together with a time stamping mechanism.

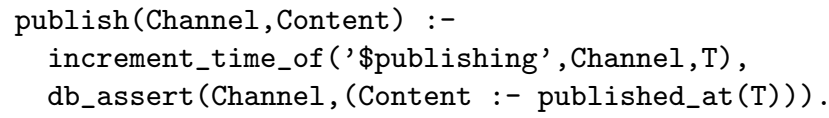

Content is a fact to be added to the database, for which the user can (and should) provide indexing declarations to support scalable large volume associative search.

The predicate consume_new (Subscriber, Channel, Content) reads the next message on Channel. It ensures, by checking and updating channel and subscriberspecific time stamps, that on each call it gets one new event, if available.

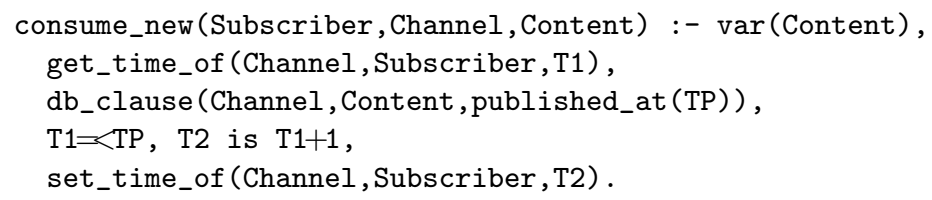

The predicate peek_at_published(ContentPattern, Matches) supports associative search for published content, independently of the fact that it has already been read. It provides to an agent the set of subscribed events matching ContentPattern.

peek_at_published(Channel, ContentPattern, Matches) :findall (ContentPattern, db_clause (Channel, ContentPattern,_), Matches).

The predicate init_publishing (ContentIndexings) sets up indexing using list of ContentIndexings of the form pred (I1, I2, . . In) where I1, I2 . . In can be 1 (indicating that an argument should be indexed) or 0 . 


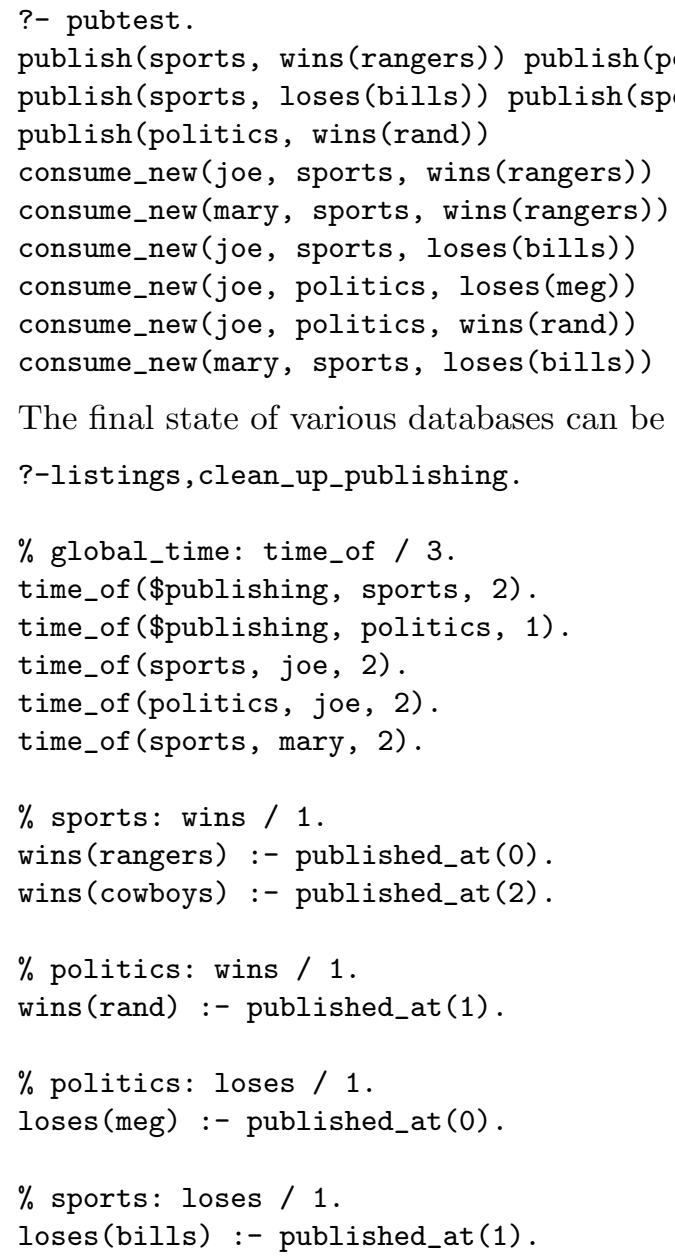

The final state of various databases can be queried (and cleaned up) with:

This shows the presence of sequencing information provided by the dynamic predicate published_at/1. Note also that these (indexed) databases can be searched associatively by various subscribers for "past" content.

\section{Related work}

Multiple logic engines have been present in one form or another in various parallel implementation of logic programming languages, $[15,16]$ Among the earliest examples of parallel execution mechanisms for Prolog, AND-parallel [7] and ORparallel [17] execution models are worth mentioning.

However, with the exception of the author's papers on this topic $[8,18,10$, 9] there are relatively few examples of using first-class logic engines as a mechanism to enhance language expressiveness, independently of their use for parallel programming. A notable exception is [19] where such an API is discussed for parallel symbolic languages in general. 
In combination with multithreading, our own engine-based API bears similarities with various other Prolog systems, notably [12,13]. Coroutining has also been present in logic languages to support constraint programming extensions requiring suspending and resuming execution based on changes of the binding state of variables. In contrast to these mechanisms that focus on transparent, fine-grained coroutining, our engine-based mechanisms are coarse-grained and programmer controlled. Our coroutining constructs can be seen, in this context, as focussed on expressing cooperative design patterns that typically involve the use of a procedural multi-threading API.

Finally, our multi_findall and multi_fold predicates have similarities with design patterns like ForkJoin [20] or MapReduce [21] coming from sharing a common inspiration source: higher-order constructs like map and fold in functional programming.

\section{Conclusion}

We have shown that by decoupling logic engines and threads, programming language constructs for coordination can be kept simple when their purpose is clear - multi-threading for performance is separated from concurrency for expressiveness. This is achieved via communication between independent language interpreters independent of the multi-threading API.

Our language constructs are particularly well-suited to take advantage of today's multi-core architectures where keeping busy a relatively small number of parallel execution units is all it takes to get predictable performance gains, while reducing the software risks coming from more complex concurrent execution mechanisms designed with massively parallel execution in mind.

The current version of LeanProlog containing the implementation of the constructs discussed in this paper, and related papers describing other aspects of the system are available at http://logic.cse.unt.edu/tarau/research/LeanProlog .

\section{Acknowledgment}

We thank NSF (research grant 1018172) for support.

\section{References}

1. Tarau, P.: Towards Inference and Computation Mobility: The Jinni Experiment. In Dix, J., Furbach, U., eds.: Proceedings of JELIA'98, LNAI 1489, Dagstuhl, Germany, Springer (October 1998) 385-390 invited talk.

2. Tarau, P.: Intelligent Mobile Agent Programming at the Intersection of Java and Prolog. In: Proceedings of The Fourth International Conference on The Practical Application of Intelligent Agents and Multi-Agents, London, U.K. (1999) 109-123

3. Tarau, P.: Inference and Computation Mobility with Jinni. In Apt, K., Marek, V., Truszczynski, M., eds.: The Logic Programming Paradigm: a 25 Year Perspective, Springer (1999) 33-48 ISBN 3-540-65463-1. 
4. Tarau, P.: Agent Oriented Logic Programming Constructs in Jinni 2004. In Demoen, B., Lifschitz, V., eds.: Logic Programming, 20-th International Conference, ICLP 2004, Saint-Malo, France, Springer, LNCS 3132 (September 2004) 477-478

5. Mayfield, J., Labrou, Y., Finin, T.W.: Evaluation of KQML as an Agent Communication Language. In Wooldridge, M., Müller, J.P., Tambe, M., eds.: ATAL. Volume 1037 of Lecture Notes in Computer Science., Springer (1995) 347-360

6. FIPA: FIPA 97 specification part 2: Agent communication language (October 1997) Version 2.0.

7. Hermenegildo, M.V.: An abstract machine for restricted and-parallel execution of logic programs. In: Proceedings on Third international conference on logic programming, New York, NY, USA, Springer-Verlag New York, Inc. (1986) 25-39

8. Tarau, P.: Fluents: A Refactoring of Prolog for Uniform Reflection and Interoperation with External Objects. In Lloyd, J., ed.: Computational Logic-CL 2000: First International Conference, London, UK (July 2000) LNCS 1861, Springer-Verlag.

9. Tarau, P., Majumdar, A.: Interoperating Logic Engines. In: Practical Aspects of Declarative Languages, 11th International Symposium, PADL 2009, Savannah, Georgia, Springer, LNCS 5418 (January 2009) 137-151

10. Tarau, P.: Logic Engines as Interactors. In Garcia de la Banda, M., Pontelli, E., eds.: Logic Programming, 24-th International Conference, ICLP, Udine, Italy, Springer, LNCS (December 2008) 703-707

11. Tarau, P., Boyer, M.: Nonstandard Answers of Elementary Logic Programs. In Jacquet, J., ed.: Constructing Logic Programs. J.Wiley (1993) 279-300

12. Carro, M., Hermenegildo, M.V.: Concurrency in Prolog Using Threads and a Shared Database. In: ICLP. (1999) 320-334

13. Wielemaker, J.: Native Preemptive Threads in SWI-Prolog. In Palamidessi, C., ed.: ICLP. Volume 2916 of Lecture Notes in Computer Science., Springer (2003) $331-345$

14. De Bosschere, K., Tarau, P.: Blackboard-based Extensions in Prolog. Software Practice and Experience 26(1) (January 1996) 49-69

15. Gupta, G., Pontelli, E., Ali, K.A., Carlsson, M., Hermenegildo, M.V.: Parallel execution of prolog programs: a survey. ACM Trans. Program. Lang. Syst. 23(4) (2001) 472-602

16. Shapiro, E.: The family of concurrent logic programming languages. ACM Comput. Surv. 21(3) (1989) 413-510

17. Lusk, E., Mudambi, S., Gmbh, E., Overbeek, R.: Applications of the aurora parallel prolog system to computational molecular biology. In: In Proc. of the JICSLP'92 Post-Conference Joint Workshop on Distributed and Parallel Implementations of Logic Programming Systems, Washington DC, MIT Press (1993)

18. Tarau, P., Dahl, V.: High-Level Networking with Mobile Code and First Order AND-Continuations. Theory and Practice of Logic Programming 1(3) (May 2001) 359-380 Cambridge University Press.

19. Casas, A., Carro, M., Hermenegildo, M.: Towards a high-level implementation of flexible parallelism primitives for symbolic languages. In: PASCO '07: Proceedings of the 2007 international workshop on Parallel symbolic computation, New York, NY, USA, ACM (2007) 93-94

20. Lea, D.: A Java fork/join framework. In: Proceedings of the ACM 2000 conference on Java Grande. JAVA '00, New York, NY, USA, ACM (2000) 36-43

21. Lämmel, R.: Google's MapReduce programming model revisited. Sci. Comput. Program. 68 (October 2007) 208-237 